\section{Kinh tế và Dụ̂ báo}

ECONOMY AND FORECAST REVIEW

Cơ QUAN NGÔN LUẬN CỦA Bộ KẾ HOẠCH VÀ ĐẦU TƯ

Tổng Biên tập

ThS. NGUYỄN LÊ THỦY

Phó Tổng Biên tập

ThS. ĐỖ TH! PHƯƠNG LAN

Hội đồng Biên tập

TS. CAO VIẾT SINH

PGS, TS. LÊ QUỐC LÝ

PGS, TS. BÙI TẤT THẮNG

TS. NGUYỄN ĐìNH CUNG

PGS, TS. NGUYỄN HỒNG SOON

GS, TS. TRẦN THOQ ĐẠT

PGS, TS. TRẦN ĐİNH THIÊN

PGS, TS. NGUYỄN ĐìNH THỌ

PGS, TS. NGUYỄN TIÉN DŨNG

TS. VƯOONG QUÂN HOÀNG

Tòa soạn và trị sự

65 Văn Miếu - Đống Đa - Hà Nội

Tel: 080.43174 / 080.49422

Fax: 024.3747.3357

Email: kinhtedubao@mpi.gov.vn

Tạp chí điện tử

http://kinhtevadubao.vn

Quảng cáo và phát hành

Tel: 080.44474 / 0909869868

Qua mạng lưới Bưu điện Việt Nam

Giấy phép xuất bản: 115/GP-BTTTT

In tại: Công ty CP in Công đoàn Việt Nam

Giá 25.000 đồng
MUC LUC

Triệu Tất Đạt: Sự khác biệt của tăng năng suất, tiến bộ kỹ thuật và thay đổi hiệu quả trong ngành chế biến, chế tạo theo cấu trúc sở hữu..................................................................... 3 Nguyễn Thị Tuyết Nhung: Đánh giá đóng góp của các yếu tố đến tăng trưởng năng suất lao động của Việt Nam giai đoạn 2011-2019........................................................................ 8 Nguyễn Thị Hồng Nhâm, Trần Minh Hiêuu: An toàn vốn của các ngân hàng thương mại ở Việt Nam.

Đỗ Hoài Linh, Nguyê̂n Thị Thảo, Đinh Thanh Tú, Hoàng Phương Hoa, Phạm Hoàng Phương Mai, Nguyển Đức Thành: Các nhân tố ảnh hưởng tới tính hữu hiệu của hoạt động kiểm soát tín dụng xanh tại các ngân hàng thương mại Việt Nam .......................................... 16 Phạm Ngọc Khanh: Phân tích mức độ ảnh hưởng của các nhân tố đến chất lượng tăng trưởng kinh tế của vùng Kinh tế trọng điểm phía Nam.....

Lê Huy Đức, Trần Thị Ngọc Anh, Nguyễn Thị Lộc An, Nguyễn Thị Ngọc Bích, Đinh Thái Hà, Vũ Thị Hằng: Tác động của các nhân tố thích ứng công việc đến sự hài lòng của các doanh nghiệp đối với sinh viên mới tốt nghiệp

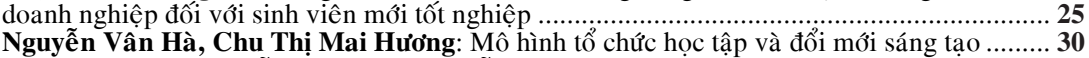
Lương Thu Hà, Nguyễn Mai Phương, Đỗ Thu Hương: Đánh giá thành công của Việt Nam trong việc kiểm soát dich Covid-19 dưới góc độ hành vi của người dân .................................. 34 Lê Ngọc Danh, Nguyễn Thị Mỹ Linh: Phân tích tài chính và các yếu tố ảnh hưởng đến sản lượng mô hình nuôi tôm tự nhiên tại xã Bình An, huyện Kiên Lương, tỉnh Kiên Giang .......... 38 Hà Nam Khánh Giao, Võ Ngọc Lê: Ảnh hưởng của văn hóa doanh nghiệp đến sự gắn bó của nhân viên Công ty TNHH Dây cáp điện Tai Sin Vietnam

Nguyễn Anh Tuấn, Vũ Thị Phương, Nguyễn Thị Quỳnh Trang, Nguyễn Thị Đào,

Tô Thế Nguyên: Tác động của dịch Covid-19 tới hoạt động học tập của sinh viên trên địa bàn TP. Hà Nôi

Nghiêm Phúc Hiếu, Trang Tử Tư Thành, Trần Văn Thi, Trần Thu Trang, Trần Tây Sơn, Nguyễn Thanh Hoa, Trần Lê Đình Hiếu: Các nhân tố ảnh hưởng đến tài sản thương hiệu bánh tráng An Ngãi, huyện Long Điền, tỉnh Bà Rịa - Vũng Tàu .....

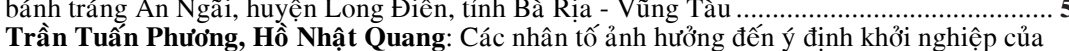

sinh viên Trường Đai hoc Khoa hoc tư nhiên, Đai hoc Quốc gia TP. Hồ Chí Minh....

Đỗ Anh Tài, Đố Đình Long, Đoà̀n Mạnh Hồng: Thực trạng thất nghiệp và công tác

tạo việc làm cho người lao động sau khi bị thất nghiệp từ các doanh nghiệp trên địa bàn tỉnh Vĩnh Phúc

Lê Thu Hạnh: Các học thuyết áp dụng cách tiếp cận nội dung trong nghiên cứu động lực làm việc

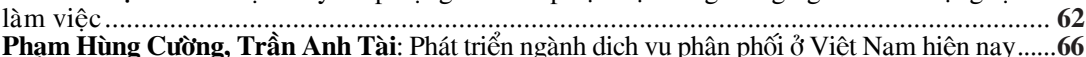
Vũ Đức Năng, Phạm Cảnh Huy: Các nhân tố tác động đến phát triển doanh nghiệp công nghiệp hỗ trơ trên đia bàn TP. Hà Nôi

Lê Văn Nam, Kiều Thị Kim Giang, Trần Ngocc Huyền, Nguyễn Thị Thu Trang: Các nhân tô. tác động đến hành vi sử dụng mạng xã hội TikTok của học sinh trung học phổ thông tại Hà Nội ......75 Nguyễn Ngọc Dương: Đánh giá sự hài lòng về chất lượng dịch vụ thương mại của các doanh nghiệp phân phối hàng hóa tai Lào Cai ....

Thái Trần Hiền Trang, Đỗ Phương Tháo, Nguyễn Thị Việt Nga: Các yếu tố tác động đến nhận thức rủi ro về thiên tai của các hộ gia đình tại Quảng Bình......................................... 84 Đoàn Thị Thu Trang: So sánh ý định khởi nghiệp của sinh viên các khối ngành kỹ............. - Nghiên cứu tại Trường Đại học Bách khoa Hà Nội.............................................................. 88

Phan Thị Minh Lý: Đánh giá tác động của vốn vay Ngân hàng Chính sách xã hội đến mức sống của hộ nghèo ở các huyện thuộc Hà Nội .......................................................................................... 92 Nguyễn Văn Anh, Phan Tuấn Anh: Các nhân tố tác động đến ý định tiếp nhận marketing qua các công cụ truyền thông xã hội

Pham Mỹ Hằng Phương: Xác định chỉ số phát triển toàn diện tỉnh Vĩnh Phúc.......................100

Lê Thị Quỳnh Nhung: Các nhân tố tác động đến kết quả học tập của sinh viên trong

bối cảnh học trực tuyển - Nghiên cứu thực nghiệm từ Học viện Ngân hàng ..........................105

Trịnh Thu Thừ, Nguyễn Thị Bích Nguyệt: Nhận thức và thái độ của giới trẻ TP. Hà Nội

đối với sản phẩm thân thiện môi trường .............................................................................109

Nguyễn Thị Hoàng Oanh: Động cơ thúc đẩy tham gia mua sắm trên mạng xã hội của nhân viên văn phòng tại TP. Hồ Chí Minh...

Nguyễn Như Ý, Phạm Văn Cà, Phạm Quốc Khởi, Huỳnh Thị Mỹ Hương, Lề Thị Huế

Trâm: Nhận diện ảnh hưởng rủi ro công nghệ thông tin đến chẩt lượng hệ thống thông tin

kế toán tại các trường học trên địa bàn tỉnh Trà Vinh ..........................................................116

Nguyễn Giác Trí: Đề xuất thang đo các nhân tố ảnh hưởng đến động lực học tập của.................

Trường Đại học Đồng Tháp....................................................................................................... 12

Huỳnh Thị Thu Sương, Nguyễn Văn Dành: Nhu câ̂u nhân lực trong các khu công nghiệp

tỉnh Bình Dương và cách thức các doanh nghiệp duy trì nguồn nhân lực ..............................123

Ngô Minh Tâm, Nguyễn Tài Năng, Ngô Minh Tiến: Các yếu tố ảnh hưởng đến ý định

mua sản phẩm xanh của khách hàng tại các siêu thị ở TP. Huế, tỉnh Thừa Thiên Huế ..........128

Trần Kim Tú, Phạm Văn Tài: Các yếu tố ảnh hưởng đến sự hài lòng của bệnh nhân về

dịch vụ KCB tại Cổng ty Cổ phần Bệnh viện Đa khoa Hoàn Mỹ Minh Hải .........................132

Dương Thị Huỳnh Như: Nghiên cứu tác động của Vốn xã hội đến Ý định khởi nghiệp của

sinh viên ngành kinh tế, Trường Đại học An Giang .......................................................136

Trần Ngọc Tú: Mối quan hệ giữa Niềm tin khách hàng, Truyền miệng và Sự hài lòng của

khách hàng đối với vàng trang sức Việt Nam................................................................140

Vũ Văn Đông, Trần Văn Dung: Các yếu tố ảnh hưởng đến ý định của du khách nội địa tiếp tục

quay lại chọn dịch vụ du lịch lữ hành tại Công ty Cổ phâ̂n Du lịch tỉnh Bà Rịa - Vũng Tàu ......144

Phan Ânh Tú, Liễu Quốc Kháng: Nghiên cứu tác động của lòng tin tổ chức đến sự gắn kết

tổ chức của viên chức trên địa bàn TP. Cần Thơ ........................................................148 


\title{
Ảnh hưởng của văn hóa doanh nghiệp đến sự gắn bó của nhân viên Công ty TNHH Dây cáp điện Tai Sin Vietnam
}

HÀ NAM KHÁNH GIAO* VÕ NGỌC LE $\hat{E}^{* *}$

Tóm tắt

Nghiên cứu kiểm định các yếu tố Văn hóa doanh nghiệp (VHDN) ảnh hưởng đến sự gắn bó của nhân viên Công ty TNHH Dây cáp điện Tai Sin Vietnam (Tai Sin), bằng việc khảo sát 282 nhân viên. Kết quả cho thấy, mức độ ảnh hưởng của 6 yếu tố VHDN theo mức độ giảm dần đến Sư gắn bó của nhân viên gồm: Làm việc nhóm; Định hướng về kế hoạch tương lai; Giao tiếp trong tổ chức; Đào tạo và phát triển; Phần thưởng và công nhận; Sáng tạo trong công việc. Nghiên cứu đề ra một số hàm ý quản trị đến các nhà quản lý Công ty Tai Sin nhằm tăng cuờng sự gắn bó của nhân viên.

Từ khóa: văn hóa doanh nghiệp, sụ gắn bó của nhân viên, Công ty TNHH Dây cáp điện Tai Sin Vietnam

\begin{abstract}
Summary
This research assesses the effect of components of corporate culture on employee commitment to Tai Sin Electric Cables VN Co. Ltd. (Tai Sin) through interviewing 282 staffs. The result points out six components arranged in decreasing order of influence, which are Team work; Future orientation; Communication; Training and development; Reward and recognition; Creativity at work. From this finding, some suggestions are made for Tai Sin's managers to enhance employee commitment.
\end{abstract}

Keywords: corporate culture, employee commitment, Tai Sin Electric Cables VN Co. Ltd

\section{GIỚI THIỆU}

VHDN được coi là một triết lý quản trị, cách thức quản trị của tổ chức để cải thiện hiệu quả thực hiện công việc cũng như khả năng tác động đến tư tưởng, tình cảm và hoạt động giao tiếp trong tổ chức.

Công ty TNHH Dây cáp điện Tai Sin Vietnam thành lập năm 2006, đang đối mặt với sự lôi kéo nguồn nhân lực từ các đối thủ cạnh tranh trong và ngoài tỉnh Bình Dương (tỷ lệ nghỉ việc năm 2019 tăng đến 14,5\%), việc duy trì nguồn nhân lực ổn định, đặc biệt là nhân viền giỏi, tài năng là một nhiệm vụ rất quan trọng. Vấn đề làm thế nào tận dụng VHDN để giữ chân và tăng sự gắn bó của nhân viên với tổ chức, đặc biệt là đối với những nhân sự có chất lượng, đã trở nền cần thiết.

\section{CƠ SỞ LÝ THUYẾT VÀ MÔ HİNH NGHIÊN CứU}

\section{Cơ sở lý thuyết}

Văn hóa doanh nghiệp

VHDN có thể được mô tả như một tập hợp chung các tín ngưỡng, thông lệ, hệ thống giá trị, quy chuẩn hành vi ứng xử và cách kinh doanh riêng của từng doanh nghiệp, những mặt trên sẽ quy định mô hình hoạt động riêng của công ty và cách ứng xử của các thành viên trong tổ chức (Tunstall, 1983, trích bởi Trương Hoàng Lâm và Đỗ Thị Thanh Vinh, 2012). Luthans (1992) định nghĩa, VHDN bao gồm các chuẩn mực đạo đức, hệ thống giá trị, niềm tin và bầu không khí tại môi trường làm việc của công ty.

Theo Schein (2010) và Hà Nam Khánh Giao (2010), VHDN là mô hình về các ngầm định cơ bản chung mà các thành viên tích lũy được trong quá trình giải quyết các vấn đề thích ứng với bên ngoài và sự hòa hợp với bên trong; họ đủ khả năng nhận biết được sự phù hợp và họ sẽ hướng dẫn cho các thành viên mới cách phù hợp để nhận thức, để suy nghĩ và cảm nhận mối liên quan đến các vấn đề này.

Gắn bó với tổ chức

Theo Meyer và Allen (1991), gắn bó

*PGS, TS., Trưởng khoa Vận tải Hàng không, Học viện Hàng không Việt Nam

** Công ty TNHH Dây cáp điện Tai Sin Vietnam

Ngày nhận bài: 06/4/2021; Ngày phản biện: 10/5/2021; Ngày duyệt đăng: 17/5/2021 
với tổ chức được xem là một trạng thái tâm lý là đặc điểm của mối quan hệ nhân viên với tổ chức này và có ý nghĩa quyết định để tiếp tục hoặc chấm dứt thành viên trong tổ chức. Sự gắn bó của nhân viên với tổ chức được đánh giá dựa trên niềm tin của họ vào tổ chức, sự chấp nhận các mục tiêu và giá trị của tổ chức, sự sẵn sàng làm việc hết mình vì tổ chức và mong muốn trở thành thành viên của tổ chức.

Có nhiều nghiên cứu quan tâm đến sự gắn bó của nhân viên với tổ chức (Giao và cộng sự, 2020a, 2020b; Hà Nam Khánh Giao và cộng sự, 2020; Hà Nam Khánh Giao và Nguyễn Đặng Huyền Trân, 2017; Hà Nam Khánh Giao và Lê Trần Tấn Tài, 2016). Nghiên cứu của Tariq và cộng sự (2011) khám phá rằng, các công ty có văn hóa trong công việc có lực lượng nhân sự gắn bó hơn. Sự phù hợp giữa VHDN và nhân viên sẽ làm tăng sự gắn bó của nhân viên đối với tổ chức (Lee và Joyce, 2014).

\section{Mô hình nghiên cứu đề xuất}

Mô hình nghiên cứu được đề nghị trong bài viết này là mô hình $\mathrm{VHDN}$ của Lau và Iris (2001) được phát triển từ mô hình của Recardo và Jolly (1997), có tham khảo kết quả nghiên cứu của Giao (2019), Hà Nam Khánh Giao và Bùi Thúy An (2017) với 4 yếu tố tác động như sau: Gắn bó của nhân viên với Tai Sin $=\mathrm{f}\{$ Giao tiếp trong tổ chức, Đào tạo và phát triển, Phần thưởng và sự công nhận, Làm việc nhóm, Sự sáng tạo trong công việc, Định hướng về kế hoạch tương lai\}

$H_{l}(+)$ : Giao tiếp trong tổ chức ảnh hưởng tích cực đến sự gắn bó của nhân viên với Tai $\operatorname{Sin}$.

$H_{2}(+)$ : Đào tạo và phát triển ảnh hưởng tích cực đến sự gắn bó của nhân viên với Tai Sin.

$H_{3}(+)$ : Phần thưởng và công nhận ảnh hưởng tích cực đến sự gắn bó của nhân viên với Tai $\operatorname{Sin}$.

$H_{4}(+)$ : Làm việc nhóm ảnh hưởng tích cực đến sự gắn bó của nhân viên với Tai Sin.

$H_{5}(+)$ : Sự sáng tạo trong công việc ảnh hưởng tích cực đến sự gắn bó của nhân viên với Tai Sin.

$H_{6}(+)$ : Định hướng về kế hoạch tương lai ảnh hưởng tích cực đến sự gắn bó của nhân viên với Tai Sin.

\section{Mẫu nghiên cứu}

Khảo sát nhân viên đang làm việc tại Tai Sin được thực hiện bằng cách gửi bảng câu hỏi trực tiếp. Tổng số bảng câu
BẢNG 1: THỐNG KÊ CRONBACH'S ALPHA

\begin{tabular}{|l|c|c|r|r|}
\hline \multicolumn{1}{|c|}{ Thang đo } & $\begin{array}{c}\text { Ký } \\
\text { hiệu }\end{array}$ & $\begin{array}{c}\text { Số biến } \\
\text { quan sát }\end{array}$ & $\begin{array}{c}\text { Cronbach's } \\
\text { Alpha }\end{array}$ & $\begin{array}{c}\text { Tương quan biến } \\
\text { tổng nhỏ nhất }\end{array}$ \\
\hline Giao tiếp trong tổ chức & GTTC & 5 & 0,911 & 0,745 \\
\hline Đào tạo và phát triển & DTPT & 6 & 0,905 & 0,694 \\
\hline Phần thưởng và sự ghi nhận & PTCN & 5 & 0,845 & 0,575 \\
\hline Làm việc nhóm & LVN & 6 & 0,878 & 0,611 \\
\hline Sư sáng tạo trong công việc & STCV & 5 & 0,883 & 0,684 \\
\hline Định hướng về kế hoạch tương lai & DHKH & 5 & 0,945 & 0,782 \\
\hline Gắn bó với Tai Sin & GBTS & 5 & 0,862 & 0,619 \\
\hline
\end{tabular}

BẢNG 2: KẾT QUẢ PHÉP XOAY NHÂN TỐ CÁC BIẾN ĐộC LẬP

\begin{tabular}{|c|c|c|c|c|c|c|}
\hline \multirow{2}{*}{ Biến quan sát } & \multicolumn{6}{|c|}{ Nhân tố } \\
\hline & 1 & 2 & 3 & 4 & 5 & 6 \\
\hline DTPT, & 0,867 & & & & & \\
\hline DTPT & 0,840 & & & & & \\
\hline $\mathrm{DTPT}^{6}$ & 0,797 & & & & & \\
\hline DTPT & 0,786 & & & & & \\
\hline $\mathrm{DTPT}_{2}^{3}$ & 0,769 & & & & & \\
\hline $\mathrm{DTPT}_{4}^{2}$ & 0,754 & & & & & \\
\hline $\mathrm{DHKH}_{4}$ & & 0,856 & & & & \\
\hline $\mathrm{DHKH}_{2}$ & & 0,855 & & & & \\
\hline $\mathrm{DHKH}^{3}$ & & 0,851 & & & & \\
\hline $\mathrm{DHKH}$ & & 0,825 & & & & \\
\hline $\mathrm{DHKH}^{2}$ & & 0,821 & & & & \\
\hline $\mathrm{LVN}$ & & & 0,820 & & & \\
\hline $\mathrm{LVN}^{6}$ & & & 0,818 & & & \\
\hline $\mathrm{LVN}^{+}$ & & & 0,775 & & & \\
\hline $\mathrm{LVN}^{\mathrm{s}}$ & & & 0,746 & & & \\
\hline $\mathrm{LVN}^{4}$ & & & 0,726 & & & \\
\hline $\mathrm{LVN}_{3}^{3}$ & & & 0,710 & & & \\
\hline GTTC $_{1}$ & & & & 0,845 & & \\
\hline GTTC $^{1}$ & & & & 0,815 & & \\
\hline $\mathrm{GTTC}_{4}$ & & & & 0,802 & & \\
\hline $\mathrm{GTTC}^{4}$ & & & & 0,791 & & \\
\hline $\mathrm{GTTC}_{2}$ & & & & 0,767 & & \\
\hline $\mathrm{STCV}_{2}^{2}$ & & & & & 0,836 & \\
\hline $\mathrm{STCV}^{3}$ & & & & & 0,816 & \\
\hline STCV $^{6}$ & & & & & 0,808 & \\
\hline $\mathrm{STCV}_{\text {, }}$ & & & & & 0,786 & \\
\hline $\mathrm{STCV}^{2}$ & & & & & 0,775 & \\
\hline $\mathrm{PTCN}_{4}^{5}$ & & & & & & 0,826 \\
\hline $\mathrm{PTCN}^{4}$ & & & & & & 0,825 \\
\hline PTCN & & & & & & 0,824 \\
\hline $\mathrm{PTCN}_{2}^{3}$ & & & & & & 0,745 \\
\hline $\mathrm{PTCN}^{2}$ & & & & & & 0,690 \\
\hline Eigenvalue & 8,482 & 3,687 & 3,370 & 2,989 & 2,066 & 1,820 \\
\hline Phuơng sai trich & 26,505 & 11,552 & 10,531 & 9,342 & 6,457 & 5,687 \\
\hline Phuơng sai trich tích lũy (\%) & 26,505 & 38,027 & 48,559 & 57,901 & 64,358 & 70,044 \\
\hline
\end{tabular}

Nguồn: Kết quả phân tích dữ liệu

hỏi phát ra là 247 bảng, thu về 233 , sau khi sàng lọc, có 225 bảng khảo sát hợp lệ được sử dụng để làm dữ liệu cho nghiên cứu, đạt tỷ lệ 86,52\%.

\section{KẾT QUẢ NGHIÊN CỨU}

\section{Đánh giá độ tin cậy của thang đo}

Kết quả kiểm định thang đo Cronbach's Alpha cho thấy các thang đo đều đạt độ tin cậy (Bảng 1). Các hệ số tương quan biến tổng đều đạt yêu cầu $(>0,3)$ và Cronbach's Alpha thấp nhất là 0,845 (Hà Nam Khánh Giao và Bùi Nhất Vương, 2019). 37 biến quan sát còn lại (sau khi loại $\mathrm{STCV}_{4}$ ) sẽ được sử dụng trong phân tích nhân tố khám phá (EFA).

\section{Phân tích EFA}

Kết quả (Bảng 2) cho thấy, $\mathrm{KMO}=0,830$ đạt yêu cầu $>0,5$ và hệ số Barlett có mức ý nghĩa Sig. $=0,000$ 
BẢNG 3: KẾT QUẢ PHÂN TícH EFA BIẾN PHỤ THUộC

\begin{tabular}{|l|r|}
\hline \multicolumn{1}{|c|}{ Biến quan sát } & \multicolumn{1}{c|}{ Nhân tố } \\
\hline GBTS $_{5}$ & 0,849 \\
\hline GBTS $_{1}$ & 0,836 \\
\hline GBTS $_{2}$ & 0,805 \\
\hline GBTS $_{3}$ & 0,769 \\
\hline GBTS $_{4}$ & 0,754 \\
\hline
\end{tabular}

BẢNG 4: TÓM TẮT MÔ HìNH HỒI QUY TUYẾN TíNH

\begin{tabular}{|c|c|c|c|c|c|c|c|}
\hline \multirow[t]{2}{*}{ Mô hình } & \multicolumn{2}{|c|}{$\begin{array}{l}\text { Hệ số } \\
\text { chưa chuẩn hóa }\end{array}$} & \multirow{2}{*}{$\begin{array}{c}\begin{array}{c}\text { Hê̂ số } \\
\text { chuẩn hóa }\end{array} \\
\text { Beta } \\
\end{array}$} & \multirow[b]{2}{*}{ t } & \multirow[t]{2}{*}{ Sig. } & \multicolumn{2}{|c|}{$\begin{array}{c}\text { Thống kê } \\
\text { đa cộng tuyến }\end{array}$} \\
\hline & B & Sai số chuẩn & & & & Dung sai & VIF \\
\hline (Hằng số) & $-0,306$ & 0,269 & & $-1,135$ & 0,258 & & \\
\hline GTTS & 0,172 & 0,049 & 0,193 & 3,513 & 0,001 & 0,682 & 1,466 \\
\hline LVN & 0,314 & 0,052 & 0,304 & 5,990 & 0,000 & 0,799 & 1,251 \\
\hline$1 \longdiv { \mathrm { PTCN } }$ & 0,116 & 0,047 & 0,113 & 2,464 & 0,014 & 0,972 & 1,029 \\
\hline DTPT & 0,159 & 0,051 & 0,157 & 3,122 & 0,002 & 0,814 & 1,228 \\
\hline STCV & 0,084 & 0,048 & 0,088 & 1,739 & 0,083 & 0,808 & 1,238 \\
\hline DHKH & 0,260 & 0,049 & 0,301 & 5,311 & 0,000 & 0,642 & 1,557 \\
\hline$R^{2}$ hiệu chinh & & & & & & & 0,539 \\
\hline Durbin-Watson & & & & & & & 1,954 \\
\hline$F$ & & & & & & & 44,665 \\
\hline Sig. & & & & & & & 0,000 \\
\hline
\end{tabular}

Nguồn: Kết quả phân tích dữ liệu

$<0,5$ đã khẳng định rằng, phương pháp phân tích trên là phù hợp. Tổng phương sai trích là $70,044 \%$ tức là 32 biến rút trích ra góp phần giải thích được khoảng $70,044 \%$ sự biến thiên của 6 biến độc lập và hệ số tải nhân số đều lớn hơn 0,5 , nên đạt yêu cầu.

Kết quả EFA biến phụ thuộc (Bảng 3) cho thấy $\mathrm{KMO}=0,838$ và hệ số Barlett có mức ý nghĩa Sig. = $0,000<0,5$, phương sai trích $64,543 \%$ và các biến đều có hệ số tải nhân tố lớn hơn 0,5 .

Phân tích hồi quy tuyến tính

Kết quả phân tích tương quan Pearson's chỉ ra rằng các hệ số tương quan đều có ý nghĩa thống kê (Sig. < $0,05)$, nên tất cả các biến sẽ được sử dụng trong phân tích hồi quy ở bước tiếp theo. Kết quả phân tích hồi quy bội như trong Bảng 4 .

Phân tích ANOVA cho thấy, kiểm định $\mathrm{F}=44,665$, Sig. < 0,05 , mô hình hồi quy tuyến tính bội đã xây dựng phù hợp với tập dữ liệu ở độ tin cậy $95 \%$. Giá trị Durbin-Watson $(\mathrm{d})=1,954$, không có tương quan giữa các phần dư. Đồng thời, VIF $<10$, không xảy ra hiện tượng đa cộng tuyến. Như vậy, mô hình hồi quy tuyến tính không vi phạm các giả định cần thiết.

Phương trình hồi quy tuyến tính bội có dạng như sau:

GBTS $=-0,306+0,172 * G T T S+0,314 * L V N$ $+0,116 * P T C N+0,159 * D T P T+0,084 * S T C V+$ $0,260 * D H K H$

\section{KẾT LUÂN VÀ HÀM Ý QUẢN TR!}

\section{Kết luận}

Sau khi tiến hành kiểm định thang đo bằng Cronbach's Alpha và phân tích EFA, mô hình nghiên cứu có 37 biến quan sát, trong đó các thang đo biến độc lập có 32 biến gồm 6 thành phần. Kết quả hồi quy đã tìm ra mối quan hệ cùng chiều giữa các yếu tố tác động đến sự gắn bó với tổ chức, sắp theo thứ tự độ mạnh giảm dần: (1) Làm việc nhóm $(\beta=0,304)$; (2) Định hướng về kế hoạch tương lai $(\beta=0,301)$; (3) Giao tiếp trong tổ chức $(\beta=0,193)$; (4) Đào tạo và phát triển $(\beta=0,157)$; (5) Phần thưởng và công nhận $(\beta=0,113)$; (6) Sáng tạo trong công việc $(\beta=0,088)$.

\section{Hàm ý quản trị}

\section{Làm việc nhóm}

Công ty cần chú trọng tổ chức các hoạt động xây dựng, phát triển đội nhóm (team building) ở trong và ngoài công ty. Xây dựng mối quan hệ trong nhóm không chỉ là giữa các nhân viên, mà còn giữa nhân viên với cấp trên. Ngoài ra, để tăng cường tinh thần làm việc nhóm, hướng đến mục tiêu chung của nhóm, của phòng/ban, tổ chức, công ty nên thực hiện chính sách đánh giá nhân viên gắn kết quả cá nhân với kết quả tập thể.

\section{Đinh hướng về kế hoach tương lai}

Những định hướng tương lai là nền tảng giúp tổ chức hoạch định các mục tiêu, kế hoạch cụ thể, phù hợp và những thay đổi cần thiết, qua đó liên kết các hoạt động của tổ chức cho các kết quả cần hướng đến. Việc chia sẻ những chiến lược, mục tiêu của doanh nghiệp giúp nhân viên hiểu rõ hơn về hoạt động của tổ chức, cảm nhận mình là một phần của tổ chức; từ đó, mỗi nhân viên nhận thức được vai trò, công việc của mình đóng góp vào thành công chung cũng như sẵn sàng nỗ lực hết mình vì tương lai của tổ chức.

\section{Giao tiếp trong tổ chức}

Người lãnh đạo phải tạo được các kênh giao tiếp có hiệu quả tích cực giữa cá nhân - cá nhân và cá nhân - lãnh đạo. Cấp lãnh đạo cần thường xuyên gần gũi, theo dõi nhân viên trong quá trình thực hiện công việc. Đồng thời, nên khuyến khích nhân viên cung cấp các thông tin đầy đủ, chính xác để cấp trên có cơ sở giải quyết những khó khăn, vướng mắc trong công việc một cách kịp thời và nhanh chóng.

\section{Đào tạo và phát triển}

Lãnh đạo Công ty cần quan tâm tổ chức các khóa đào tạo các kỹ năng cơ bản thông qua chương trình đào tạo tại chỗ, xây dựng thêm những khóa học thông dụng khác nhằm giúp nhân viên bộ phận có được các kiến thức và kỹ năng bổ sung. Bên cạnh đó, cần bổ sung thêm các chương trình đào tạo và phát triển từ bên ngoài, như: phần mềm/công cụ thiết kế ứng dụng kiến thức tiên tiến, 
khoá học về nâng cao nghiệp vụ... tạo bước chuẩn bị đội ngũ quản lý kế cận.

\section{Phần thưởng và công nhận}

Khi nhân viên thực hiện tốt công việc, thì cấp quản lý trực tiếp cần động viên, khích lệ nhân viên ngay lúc đó. Các hình thức có thể thực hiện, như: khen thưởng đột xuất, một bản nhận xét cá nhân được người quản lý gửi trực tiếp đến nhân viên; tuyên dương nhân viên này trước các đồng nghiệp, có tác động tích cực đến tổ chức...

\section{Sáng tạo trong công việc}

Để nâng cao sự sáng tạo trong công việc, Công ty không chỉ dừng lại ở mức độ động viên khuyến khích nhân viên phát triển ý tưởng mới, mà cần nghiên cứu thực hiện cải tiến quy trình tạo ra môi trường để nhân viên có thể tiếp cận với việc đề xuất ý tưởng sáng tạo.

\section{TÀI LIỆ THAM KHẢO}

1. Hà Nam Khánh Giao và Bùi Nhất Vương (2019). Giáo trình Cao học - Phương pháp Nghiên cứu khoa học trong kinh doanh - Cập nhật SmartPLS, Nxb Tài chính

2. Hà Nam Khánh Giao (2010). Mạn đàm thước đo Văn hóa Doanh nghiệp trong các Ngân hàng thương mại, Tạp chí Ngân hàng, 22(11), 25-27

3. Hà Nam Khánh Giao và Bùi Thị Thúy An (2017). Ảnh hưởng của văn hóa doanh nghiệp đến sự gắn bó của nhân viên Công ty Cổ phần Thủy sản Sóc Trăng, Tạp chí Khoa hoc Trường Đại học Đồng Tháp, 24(2), 36-40

4. Hà Nam Khánh Giao, Huỳnh Diệp Trâm Anh, Nguyễn Thị Kim Ngân, Đoàn Quang Đồng, Nguyễn Phạm Hạnh Phúc, Bùi Nhất Vương (2020). Ảnh hưởng của vốn tâm lý, tính cách cá nhân đến sự gắn kết cồng việc của nhân viên khách sạn tại TP. Hồ Chí Minh, Tạp chí Khoa học Trường Đại họ Trà Vinh, 37(3), 12-23

5. Hà Nam Khánh Giao và Lê Trần Tấn Tài (2016). Các nhân tố tác động đến gắn kết của nhân viên với tổ chức tại UFM, Tạp chí Nghiên cứu Tài chính - Marketing, 34(8), 61-67

6. Hà Nam Khánh Giao và Hồ Thị Thu Trang (2016). Ảnh hưởng của văn hóa tổ chức đến sự gắn kết của nhân viên văn phòng tại tỉnh Bà Rịa - Vũng Tàu, Tạp chí Kinh tế - Kỹ thuật Bình Dương, 14(6), 39-49

7. Hà Nam Khánh Giao và Nguyễn Đặng Huyền Trân (2017). Các yếu tố ảnh hưởng đến sự gắn kết nhân viên với Trường Đại học Ngân hàng TP. HCM, Tạp chí Công Thương, 10(9), 246-251

8. Hà Nam Khánh Giao và Bùi Nhất Vương (2016). Ảnh hưởng của các yếu tố văn hóa doanh nghiệp đến sự gắn bó của nhân viên với tổ chức tại Công ty $\mathrm{CP}$ CMC Telecom TP. HCM, Tạp chí Kinh tế - Kỹ thuật Bình Dương, 13(3), 87-101

9. Trương Hoàng Lâm và Đỗ Thị Thanh Vinh (2012). Ảnh hưởng của văn hóa doanh nghiệp đến mức độ cam kết của nhân viên: Trường hợp công ty hệ thống thông tin FPT, Tạp chí Kinh tế \& Phát triển, 185(11), 120-127

10. Giao H. N. K. (2019). The effect of corporate culture on the staff's commitment in CMC Telecome Vietnam, Science Journal of Business and Management, 7(1), 23-32, Doi: 10.11648/j.sjbm.20190701.14

11. Giao H. N. K., Vuong B. N., and Tung D. D. (2020a). A model of Organizational Culture to Enhancing Organizational Commitment in The Telecom Industry: An Evidence from Vietnam, World Scientific and Engineering Academy and Society (WSEAS) Transactions on Business and Economics, 17, 215-224, DOI: 10.37394/23207.2020.17.23

12. Giao H. N. K., Vuong B. N., Huan D. D., Tushar H., and Quan T. N. (2020b). The Effect of Emotional Intelligence on Turnover Intention and the Moderating Role of Perceived Organizational Support: Evidence from the Banking Industry of Vietnam, Sustainability, MDPI, 12(5), 1857-1882, Doi:10.3390/su12051857

13. Lau H. C., Iris M. A. (2001). Research and concepts: The soft foundation of the critical success factors on TQM implementation in Malaysia, The TQM Magazine, 13(1), 51-60

14. Lee X. L., and Joyce L. F. Y. (2014). Organizational commitment of white collar employees in damansara heights, Kuala Lumpur, Journal of Social Economics Research, 1(7), 156-168

15. Luthans F. (1992). Organizational behavior, McGraw-Hill, New York

16. Meyer J, and Allen N. (1991). A three-component conceptualization of organizational commitment. Human Resource Management Review, 1(1), 61-89

17. Recardo R., Jolly J. (1997). Organizational Culture and Teams, SAM Advanced Management Journal, 62(2), 4-7

18. Schein H. (2010). Organizational culture and leadship, Jossey- bass, USA

19. Tariq I. K., Farooq A. J, Aisha A., Muhammad B. K., and Syed T. H. (2011). Job Involvement as Predictor of Employee Commitment: Evidence from Pakistan, International Journal of Business and Management, 6(4), 252-262 